\title{
PERCEPTUAL REVERSAL GUIDED BY INTEGRATION BETWEEN BOTTOM-UP INPUT AND TOP-DOWN FEEDBACK OVER TIME COURSE
}

\author{
KangWoo LEE \\ Convergence Research Center, Sungkyunkwan University, Korea
}

\begin{abstract}
A computational model of perceptual reversal alternating between two interpretations is presented. Initially, the model represents the ambiguous state of a reversible picture, such as the bistable face-vase image. The internal state of the network evolves to settle into a stable state, which corresponds to one of two alternatives. Top-down feedback proves a deciding factor in leading the system into a modeled perceptual state over the time course. At any given time, top-down input from temporal associative memory provides contextual modulation of bottom-up input in the network. The model accounts for the role of top-down knowledge in resolving perceptual ambiguity as well as reversibility from one state to another.
\end{abstract}

Key words: Perceptual reversal, Figure-ground, Top-down feedback, Multi-stability

\section{INTRODUCTION}

A fascinating example of how attention can affect perception is polyfiguration, where a figure can be interpreted as two or more configurations, such as Escher's pictures (Ernst,1986) and Rubin's face-vase picture (Rubin, 1915). The nature of these pictures, which is reversible, allows different aspects of perceptual mechanisms to be studied, including segmentation, figure-ground organization, multi-stability, attention etc. However, most of the research is dominated by figure-ground organization, as conceptualized by Rubin (1921). The various factors that influence figure-ground relations have been investigated, for example; contrast, color, size, orientation, symmetry, binocular disparity, surroundedness etc (Palmer, 1999). However, when these factors are in equilibrium, the resulting percept is ambiguous. Questions arising at this stage are: "how can the ambiguous state be resolved?", "how does one perceptual state move to the other state?" and "what kind of mechanism is involved in multi-stable perception?" To answer these questions, we present a dynamic model with both bottom-up and top-down inputs interacting on a reversal task for an ambiguous picture. We demonstrate that the role of top-down influence is important not only to interpret and resolve the ambiguous picture, but also to stabilize the system over the time course.

In the following section, we briefly review two competing theories that explain the

This research was supported by the Basic Science Research Program (Grant no. 2012009055) through the National Research Foundation of Korea (NRF), funded by the Ministry of Education, Science and Technology.

Correspondence concerning this article should be addressed to KangWoo LEE, Convergence Research Center, Sungkyunkwan University, 300, Chon-Chon Dong, Jang-Ahn Ku, Suwon, 440-746 Korea (e-mail: kangwooster@gmail.com). 
phenomenon of perceptual reversal and computational models that address this problem. We provide the structure and mathematical aspects of the model and present a simulation of the model, with results. Regarding the psychological and neuroscientific considerations, the questions posed above will be discussed at the end of this paper.

Throughout this paper we focus on the role of top-down signals in multistable perception. However, it is obvious that both bottom-up and top-down mechanisms are involved in this fascinating phenomenon at various processing stages. Both bottom-up and top-down mechanisms are not mutually exclusive, but they cooperatively or competitively work to resolve the perceptual ambiguity.

\section{TheORETICAL AND COMPUTATIONAL EXPLANATIONS}

\section{Theoretical Explanations}

The prevailing theory to explain perceptual reversal is neural satiation or fatigue hypothesis (Long \& Toppino, 2004; Wagemans et al., 2012). In this view, reversal from one perceptual state to another state occurs if sets of neurons involved in a particular percept undergo satiation or fatigue with continuing inspection of a pattern. Decreased or fatigued neural activities in a set of neurons corresponding to one percept then lead to an alternative percept. The switch among such stable states is assumed to be automatic and spontaneous. In addition, the neural fatigue hypothesis often assumes reciprocal inhibitory connections among neurons that correspond to one of the percepts, so that as soon as one percept starts to prevail, alternatives are suppressed.

Criticizing this assumption of automatic and spontaneous reversal, the main alternative approach puts the emphasis on knowledge of ambiguity and reversibility of a given pattern (Doherty \& Wimmer, 2005). For example, Rock, Gopnik, and Hall (1994) and Rock and Mitchener (1992) have shown that many subjects fail to report spontaneous reversal if they were not informed of the possible interpretations before viewing the ambiguous pictures. Other counter-evidence comes from binocular rivalry (Balcetis, Dunning, \& Granot, 2012; Blake \& Wilson, 2011), where two eyes are presented with incongruent stimuli, such that perception alternates between each monocular view. Leopold and Logothetis (1999) comprehensively reviewed psychological and neurological aspects of binocular rivalry dealing with critical issues - the level of processing involved in the phenomenon, the brain mechanism responsible for perceptual oscillation, temporal dynamics of perceptual reversal, etc. They argued that, while the different percepts of ambiguous stimuli ultimately depend upon activity in the sensory visual area, this activity is continuously steered and modified by central brain structures involved in planning and generating behavior action. That is, top-down information from higher brain areas, such as the frontal lobe, plays a critical role in perceptual transition between alternatives. In particular, brain imaging studies on binocular rivalry have indicated that the extrastriate areas are engaged in nonrivalrous perceptual changes, while the activity in the frontal-parietal cortex is specifically associated with perceptual attention only during rivalry (Lumer, Friston, \& Rees, 1998; Sterzer, Kleinschmidt, \& Rees, 2009). 


\section{Computational Models}

Few computational models have tackled perceptual reversibility or the figure-ground separation problem. First, Rumelhart, Smolensky, McClelland, and Hinton (1986) presented a connectionist model for perception of a Necker cube. The model consists of two interconnected sub-networks that correspond to possible interpretations of the Necker cube. Each unit in each network represents the interpretation of a particular vertex in a 3D cube. For example, a unit labeled "BUR" indicates the Back Upper Right corner of a cube. Basically, the dynamics of the network are based on cooperation between consistent units and inhibition between inconsistent units. In a given interpretation, a vertex can be compared with the other three vertices that are connected to it for support. However, one interpretation of a given vertex cannot be consistent with its other interpretation. The system can end up with an interpretation of the Necker cube as either facing left or facing right. However, once it settles into one perceptual state (one of the two interpretations), it will stay in that state forever if no additional manipulation is imposed. Therefore, it does not show reversibility in a true dynamic sense.

Ditzinger and Haken (1989) illustrated a different approach on the basis of their synergetic system. In their model, an initial vector is pulled into one of the prototype patterns that has the largest overlap. They demonstrated that the use of two well-chosen parameters (attention and bias) leads to perceptual fluctuation with different time intervals. The attention parameter controlled whether or not a pattern was processed, and the bias parameter determined how long the system would pay attention to a certain pattern. Therefore, a preferentially biased pattern is attended to longer than an unpreferred pattern.

Resent approaches to perceptual reversal emphasize the role of selective attention (Craft, Schütze, Niebur, \& von der Heydt, 2007; Kawabata \& Mori, 1992) and temporal synchrony of neuronal response (Atmanspacher, Bach, Filk, Kornmeier, \& Römer, 2008; Ciszak, Euzzor, Farini, Arecchi, \& Meucci, 2012). According to the selective attentionbased approach, the parts of an object have different levels of importance. For example, the eyes and nose are more important than hair for recognizing a face. Attending to a certain part of an ambiguous picture leads the system into one of two possibilities, while attending to another part may lead to the alternative interpretation. That is, the whole image is interpreted on the basis of schemas that are obtained from selected local information. However, in these works, attention is driven purely by bottom-up information, and the switch over between two interpretations was not implemented. Thus, the system had the potential for dynamic switching but only in the attention-changing extension of the model.

Rather than individual neurons corresponding to important features or parts of a percept, the synchrony between neurons represents perceptual dominance of an alternative during the perceptual reversal. However, there is dispute over the role of synchrony in perceptual grouping (Farid, 2002; Farid \& Adelson, 2001). A recent study showed the collective activities of neural populations in bistable perception without synchrony (Gigante, Mattia, Braun, \& Del Giudice, 2009). In this scheme, sensory input is represented by distributed and collective neurons that are temporally uncoupled. These neurons gradually converge to perceptual interpretation through mutual competition between alternatives. That is, the collective experience of neurons to sensory input leads to a perceptual interpretation. 


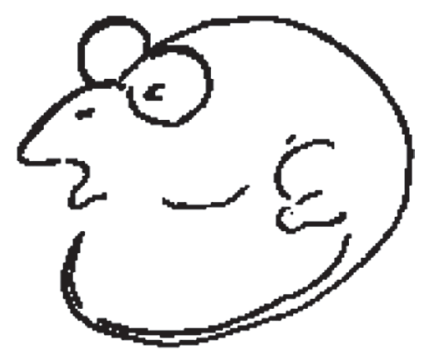

(a)

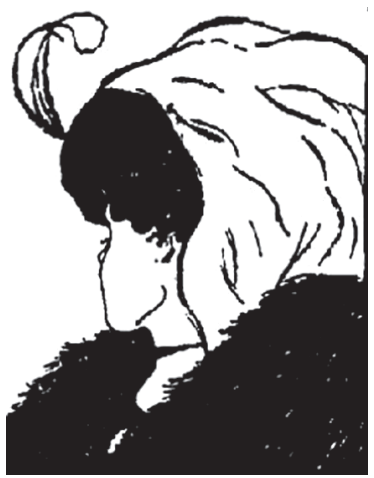

(b)

Fig. 1. Ambiguous pictures: (a) can be interpreted as a man or a rat; (b) can be interpreted as a young lady or old lady.

\section{The Proposed Model}

In this section, we present a computational model for multiperception, based on integration between bottom-up and top-down information where feedback from the higher layer stabilizes the whole system. The model essentially consists of three modules performing integration between bottom-up and top-down inputs, sequential associative memory, and feedback that leads the whole system into a certain perceptual state at a given time. However, this stable state can switch dynamically over the time course, driven by the sequential associative memory module.

\section{Architecture}

Fig. 2 shows a schematic representation of the model. The model integrates both bottom-up data coming from an input layer and top-down data from associative memory in order to produce the output. This integration module is necessary to process bottom-up input in the context of top-down input. That is, the interpretation of an ambiguous picture like the face-vase configuration depends on the top-down flow of information, since the external visual source is the same regardless of what it is perceived. Here, we may consider bottom-up input as sensory input from an external visual source and top-down input as internal representation or subjective hypothesis about the visual source. To generate integration output, the model requires two steps. (a) The net values from bottom-up and top-down inputs are processed by an activation function, and then (b) output can be obtained by applying the sigmoid function. The output values are determined by both activation and transfer functions that constrain the relationship between bottom-up and top-down inputs, based on the consistency of both inputs. Then, the outputs of the integration module are sent to the sequential (temporal) associative memory via feedforward connections.

The module for associative memory here is implemented using a Hopfield network with two different types of connections - short-term connections and long-term connections. 


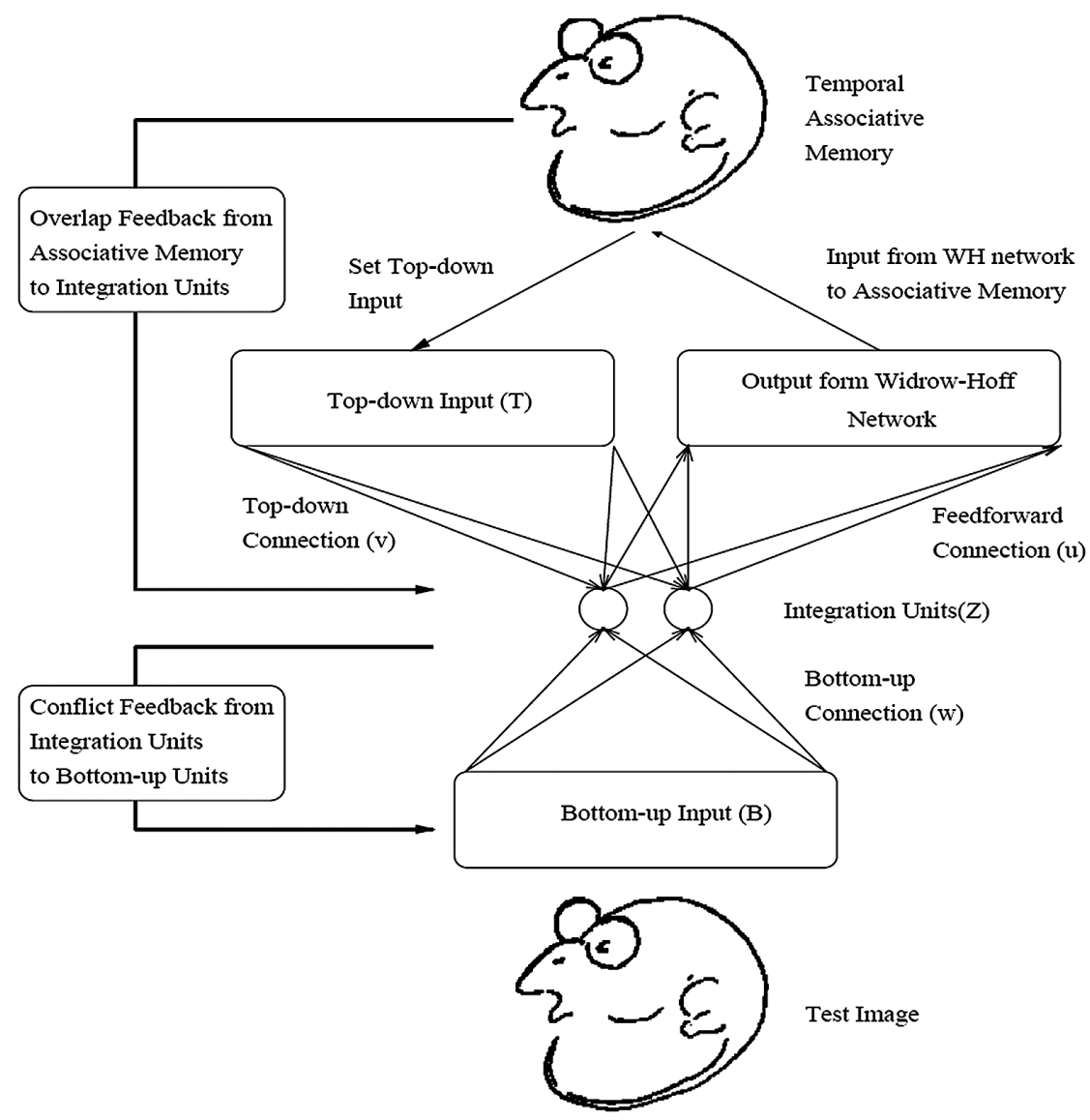

Fig. 2. Structure of the model. Basically, the model consists of three main modules - an integration module that integrates both bottom-up and top-down inputs, an associative memory module that represents inputs from an earlier stage and changes the present internal state to another state, and a feedback module that send signals in order stabilize the system.

A typical Hopfield network usually has only symmetric short-term connections that stabilize a pattern (Hopfield, 1982). In contrast, asymmetric long-term connections tend to cause transitions in the pattern sequence. Therefore, a dynamic property of the network is to move from one state to another along memorized patterns over a time course. At a specific time $t$, the state of the network can be described as the overlap of embedded pattern states (Riedel, Kühn, \& van Hemmen, 1988). For example, if two memorized patterns are simultaneously activated at differing levels, we may consider this state as an ambiguous state or transitory state. On the other hand, if only one pattern is activated at a given time, we may consider it as a stable state, which corresponds to a stored pattern, at which the network is in the local energy minimum. In addition, the output of temporal associative memory will be used as top-down input to the integration module in the next step.

The feedback module sends the overlap values to integration outputs. Here, we 
introduce the concept of conflict defined as a difference between integration output and the overlap value. This means a difference between internal representation of a higher processing stage at a time $t$ and sensory representation of a lower processing stage at the previous time $t-1$. The difference can also be thought of as corresponding to the amount of attention that should be allocated to the bottom-up input in order to resolve ambiguity. It reflects an inconsistent or unstable state of the whole system caused by discrepancy between the state of the internal representation and the state of the integration layer. Therefore, this unwanted or unstable state is minimized to settle into a consistent state. In our model, the conflict is minimized by changing the input until it reaches an acceptable criterion.

\section{Mathematical Description of the Model}

Integration Module.: The learning rule for the integration module network was first developed by Kay and Phillips (1997). The goal of the learning rule is to maximize mutual information amongst three random vectors-bottom-up input $(B U)$, top-down input $(T D)$ and integration output $(Z)$. The relationship between the three variables is described by the following equation:

$$
F=I(Z ; B U ; T D)
$$

where $I(Z ; B U ; T D)$ is the mutual information shared amongst $Z, B U$, and $T D$. The transfer function of the integration output unit is composed of two steps. The weighted inputs are used to calculate internal activation values. Then, the values are fed into a sigmoid function to obtain an integration output. For the internal activation of the $j$-th integration output unit, we first sum the bottom-up and top-down inputs:

$$
s_{j}^{B U}=\sum_{i=0}^{n} x_{i}^{B U} w_{i j}+w_{j 0} \quad s_{j}^{T D}=\sum_{k=0}^{m} x_{k}^{T D} v_{k j}+v_{k 0}
$$

where $s^{B U}$ and $s^{T D}$ are the weighted sums of bottom-up and top-down inputs, and $w$ and $v$ are weight parameters that link bottom-up input units and integration units and top-down input units and integration units, respectively. The $w_{0}$ and $v_{0}$ are biases for the inputs $x^{B U}$ and $x^{T D}$, respectively. The activation function described below integrates both weighted inputs.

$$
a_{j}=\frac{1}{2} s_{j}^{B U}\left(1+\exp \left(2 s_{j}^{B U} s_{j}^{T D}\right)\right)
$$

The sigmoid function is used to compute integration outputs.

$$
z_{j}=\frac{1}{1+\exp \left(-a_{j}\right)}
$$

Kay and Phillips (1997) have shown that the activation function has the following dynamic properties. If $s^{B U}$ is zero, then the activation will be zero; if $s^{T D}$ is zero, then the activation will be decided by $s^{B U}$; if $s^{B U}$ and $s^{T D}$ are consistent, then the gain of the transfer 
function relating to activation will be increased; if $s^{B U}$ and $s^{T D}$ are inconsistent, then the gain of the transfer function relating to activation will be decreased. To derive a learning rule, it is more convenient if $F$ in Equation 1 is expressed in terms of conditional entropies:

$$
F=H(Z)-H(Z \mid B U)-H(Z \mid T D)+H(Z \mid B U, T D)
$$

Since we wish to maximize the objective function, a gradient ascent learning rule is applied to update the weights:

$$
\Delta w_{j}=\eta \frac{\partial F}{\partial w_{j}}
$$

where $\eta$ is the learning rate, and

$$
\frac{\partial F}{\partial w_{j}}=\left\langle\left[-a_{j}-\overline{\rho_{j}}\right] z_{j}\left(1-z_{j}\right) \frac{\partial a_{j}}{\partial s_{j}^{B U}} x^{B U}\right\rangle_{B U, T D}
$$

where $\langle\ldots\rangle_{B U ; T D}$ is the average output probability over the joint distribution of $B U$ and $T D$, and where the dynamic average $\overline{\rho_{j}}$ is defined by:

$$
\overline{\rho_{j}}=\log \left(\frac{E_{j}}{1-E_{j}}\right)-\log \left(\frac{E_{j}^{B U}}{1-E_{j}^{B U}}\right)-\log \left(\frac{E_{j}^{T D}}{1-E_{j}^{T D}}\right)
$$

where $E$ is the average output probability over the joint distribution of $B U$ and $T D, E^{B U}$ is the average output probability over the conditional distribution of $T D$ given $B U=b$, and $E^{T D}$ is the average output probability over the conditional distribution of $B U$ given $T D=t$. The learning rule just specified was for the $w$ weights but is also applied to the $v$ weights, with $w$ being replaced by $v$ and $\left(\partial a_{j} / \partial s_{j}^{B U}\right) b$ being replaced by $\left(\partial a_{j} / \partial s_{j}^{T D}\right) t$,

where:

$$
\frac{\partial a_{j}}{\partial s_{j}^{B U}}=\frac{1}{2}+\left(\frac{1}{2}+s_{j}^{B U} s_{j}^{T D}\right) \exp \left(2 s_{j}^{B U} s_{j}^{T D}\right)
$$

and

$$
\frac{\partial a_{j}}{\partial s_{j}^{T}}=\left(s_{j}^{B U}\right)^{2} \exp \left(2 s_{j}^{B U} s_{j}^{T D}\right)
$$

Connections from Integration Units to Top-down Units.: To set the connections between integration units and the output units of a Widrow-Hoff network, we used a Widrow-Hoff learning rule that minimizes the output error using (approximate) gradient descent (Haykin, 1998). In the Widrow-Hoff learning rule, which produce an acceptable error, the goal is to find the set of weights for a given set of training patterns. The weight vector increment under this learning rule is:

$$
\Delta u_{k}=-\eta\left(d_{k}-o_{k}\right) z
$$


where $d$ and $o$ are the desired output and actual output, respectively. The actual output $o_{k}$ is defined as $u_{k}{ }^{t} z$.

Temporal Associative Memory.: Temporal associative memory is an associative memory for learning, storing, generating, and recalling sequences of patterns in the form of dynamic state transitions (Riedel et al., 1988). For example, a given set of sequences $\Psi$ contains $p$ vectors:

$$
\Psi=\left\{\xi^{1}, \xi^{2}, \cdots, \xi^{P}\right\}
$$

where column vector $\xi^{\mu}$ is n-dimensional. The sequence in a dynamic transition, such as $\xi^{1} \rightarrow \xi^{1} \rightarrow \ldots \rightarrow \xi^{P} \rightarrow \xi^{1} \rightarrow \xi^{2}$ should be memorized. A simple Hopfield network with only symmetric connections does not allow temporal sequence since it settles down into an attractor and stays there. To this end, the synaptic connections can be separated into two parts-short-term and long-term connections. The short-term connections are symmetric as shown in the following equation, and stabilize a pattern:

$$
c_{i j}^{S}=\frac{1}{n} \sum_{\mu} \xi_{i}^{\mu} \xi_{j}^{\mu}
$$

whereas asymmetric long-term connections

$$
c_{i j}^{L}=\frac{1}{n} \sum_{\mu} \xi_{i}^{\mu+1} \xi_{j}^{\mu}
$$

tend to cause transition between patterns. These long-term connections represent slow synapses that have delayed or sluggish responses. The net input to unit $i$ at a time $t$ is described as follows:

$$
h_{i}(t)=\sum_{j}\left[c_{i j}^{S} R_{j}(t)+\lambda c_{i j}^{L} \overline{R_{j}}(t)\right]
$$

where, if $t=1, R_{j}(t)=o_{j}(t)$, otherwise $R_{j}(t)=\varphi y_{j}(t-1)+(1-\varphi) o_{j}(t)$. The $\lambda$ is a constant that governs the relative strength of short- and long-connection terms. Here, $\varphi$ was set to 0.9 . The delay term $\bar{R}_{j}(t)$ samples the past values of $R_{j}(t)$ up to time $t$.

$$
\overline{R_{j}}(t)=\int_{-\infty}^{t} G\left(t-t^{\prime}\right) R_{j}\left(t^{\prime}\right) d t^{\prime}
$$

Various forms of a function can be used for the kernel $G(t)$. Here we used a logistic function that is used in Boltzmann machine and simulated annealing (Aarts \& Korst, 1989).

$$
G(t)=\frac{1}{1+\exp \left(-\frac{h(t)}{T}\right)}
$$

where the constant $T$ is a temperature parameter. The output of a unit in the temporal associative memory is given by: 


$$
y_{i}(t)=2 G\left(h_{i}(t)\right)-1
$$

The states of temporal associative memory are described by the overlap, calculated by multiplying training patterns and outputs of temporal associative memory.

$$
\zeta_{\mu}(t)=\frac{1}{n} \sum_{i} \xi_{i}^{\mu} y_{i}(t)
$$

where $n$ is the number of units. The overlap shows the state of each stored pattern at a given time.

Feedback Module: As referred to above, feedback in the system plays an important role in settling the whole system into a consistent or stable state. The feedback module begins by comparing overlap values with the integration output. Finally, we introduce the concept of conflict, which is defined as the difference between integration output an overlap values.

$$
C=\frac{1}{2} \sum_{j=1}^{p}\left(\zeta_{j}(t)-z_{j}(t-1)\right)^{2}
$$

This can be considered as the amount of attention that should be allocated to the bottom-up input in order to resolve any ambiguity. Therefore, the ambiguity of the stimulus should be resolved in the context of internal representation or top-down knowledge. In this case, bottom-up input can be changed in the direction that minimizes the conflict:

$$
\begin{gathered}
\Delta x_{i}^{B U}=-\eta \frac{\partial C}{\partial x_{i}^{B U}} \\
\frac{\partial C}{\partial x_{i}^{B U}}=\sum_{j=1}^{p}\left(\zeta_{j}(t)-z_{j}(t-1)\right)\left(z_{j}(t-1)-z_{j}^{2}(t-1)\right) w_{i j}\left[1+\exp \left(2 s_{j}^{B U} s_{j}^{T D}\right)\left(1+2 s_{j}^{T D}\right)\right]
\end{gathered}
$$

To minimize this conflict, the bottom-up data is changed until it reaches an acceptable criterion. It should be noted that all processes - integration, temporal associative memory, and feedback - continue in an iterative cycle.

\section{Simulation AND RESUlT}

\section{Properties of Top-down Input}

In the model, the integration unit has an important role to integrate both bottom-up and top-down inputs and produces outputs that further feed into the upper layer WH network. In particular, the function in Equation 4 has interesting properties, as shown in Fig. 3. The activation values with different $s^{T D}$ are plotted over $s^{B U}$. Using the line with $s^{T D}=0$ as a reference line, the other lines with different $s^{T D}$ are plotted. If both net values have the same sign, the lines are placed above (positive) or below (negative) the reference line. In contrast, if both net values have different signs, the lines with positive $s^{T D}$ are 


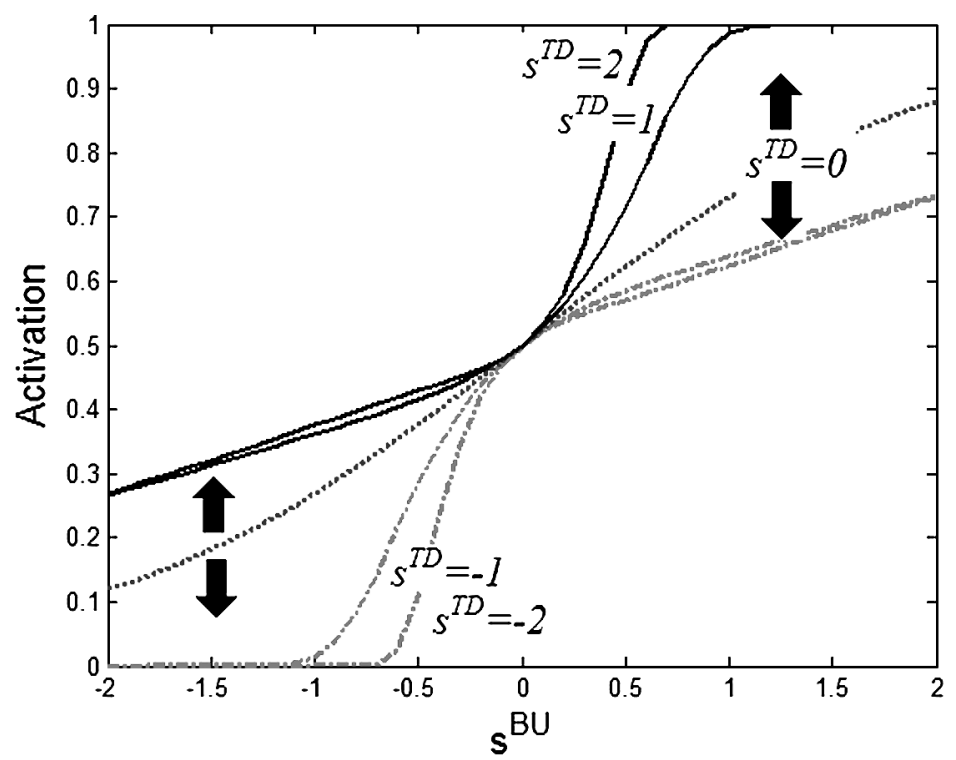

Fig. 3. The activation values with different $s^{T D}$ plotted over $s^{B U}$.

placed above the reference and the lines with negative $s^{T D}$ are placed below the reference line. So, the top-down net value $s^{T D}$ modulates the activation depending on the consistency with the bottom-up net value $s^{B U}$.

To help us further understand the role of top-down input, the temperature $T$ in Equation 17 is manipulated. In this simulation, two binary patterns $(1-1)$ and $(-11)$ were used, and the first pattern was tested. When the value of the temperature parameter $T$ was 1 , the overlap fluctuated between 1 and -1 . In contrast, when the temperature parameter $T$ was 2 or 3 , the amplitude of the overlap decreased and fluctuates toward 0 . The values of the exponential term in Equation 17 became smaller as the temperature $T$ increased. This resulted in the overlap approaching to zero over time, as shown in Fig. 4.

Interestingly, the top-down input also has similar properties shown by the temperature parameter $T$. In this simulation, the temperature parameter was fixed to 1, but the top-down input is varied by multiplications of three scalars $s c=1.0,0.5$ and 0.01 , respectively. When top-down input is multiplied by the small scalar 0.01 , the amplitude of the overlap decreases over time step and approaches 0. As shown in Fig. 3, the top-down net input should be larger than 0 , so that the integration output $z(t)$ is greater than the previous $z(t-1)$. Also, the top-down net input should be less than 0 so that the integration output $z(t)$ is smaller than the previous $z(t-1)$. Therefore, when the top-down input is set to near zero, this determines the upper and lower boundaries of overlap fluctuation. Furthermore, the change of bottom-up input that reduces the conflict $C$ in Equation 21 continuously produces integration output of 0.5 . In a similar way, damped outputs of the temporal associative memory were accumulated over time, and then approach zero.

Thus, the overlap with the top-down input close to 0, shown in Fig. 5 (c), is reduced. 


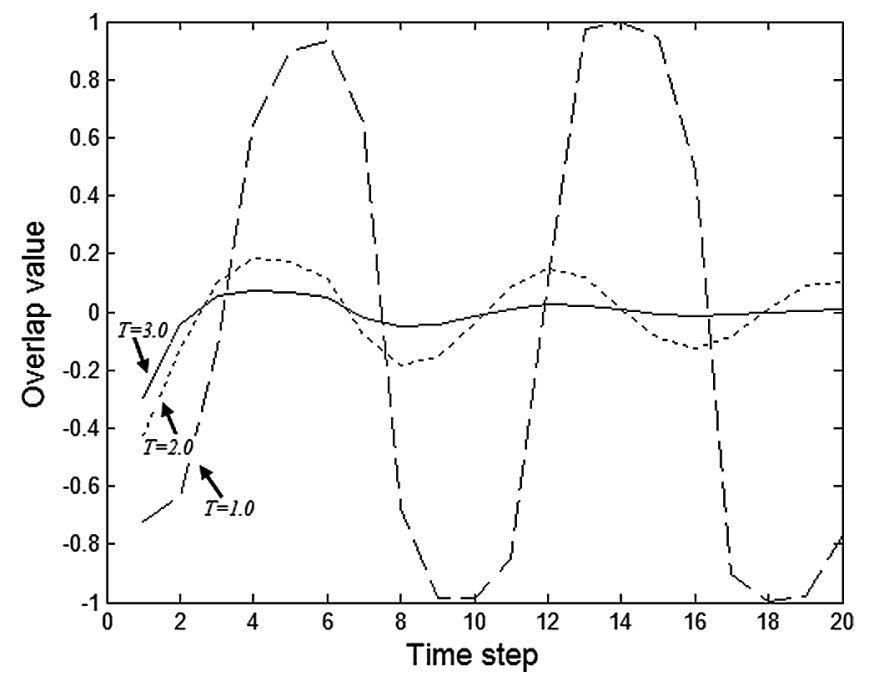

Fig. 4. The curves show the state of overlap at time $t$ with different temperature parameters $T=1, T=2$ and $T=3$.

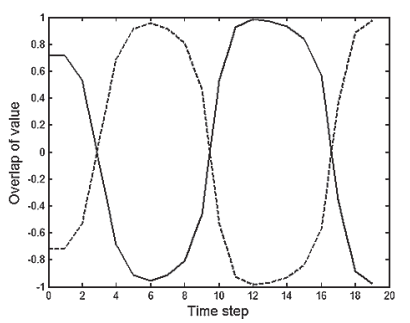

(a) $\mathrm{sc}=1.0$

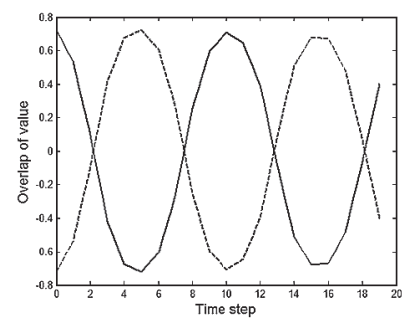

(b) $\mathrm{sc}=0.5$

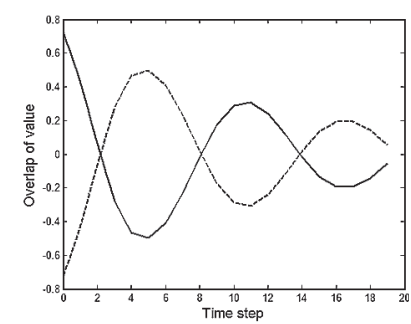

(c) $\mathrm{sc}=0.01$

Fig. 5. The overlaps with different scalars - 1.0, 0.5 and 0.01 - that multiply top-down input.

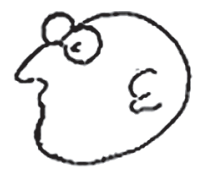

(a) Man

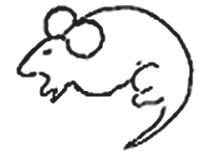

(b) Rat

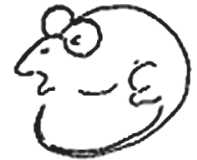

(c) Test image

Fig. 6. The man (a) and rat (b) images are used to train the model, and the ambiguous image (c) is used to test the performance of the model.

\section{Simulation of 'Man-Rat' Perceptual Reversal}

In the simulation, each module was trained individually and combined to construct a whole system. The initial weights for $w, u$, and $v$ were randomly set to low values from -0.001 to 0.001 . For the temporal associative memory, weights were calculated according to Equation 14 and Equation 15 using two image patterns - man and rat-shown in Fig. 6. 


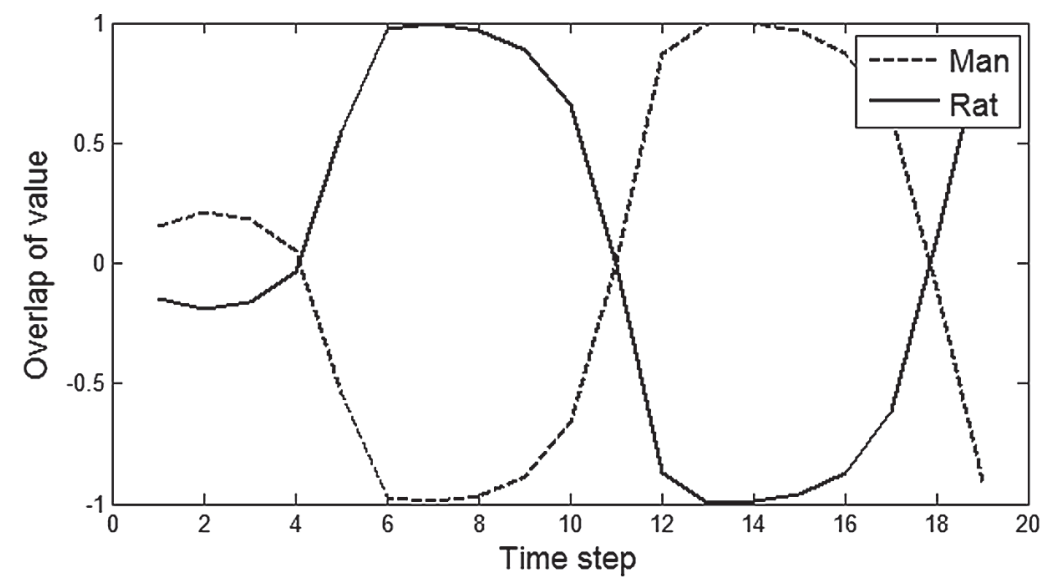

(a) The overlap states of 'man' and 'rat' perception with strong top-down feedback (sc $=1.0)$

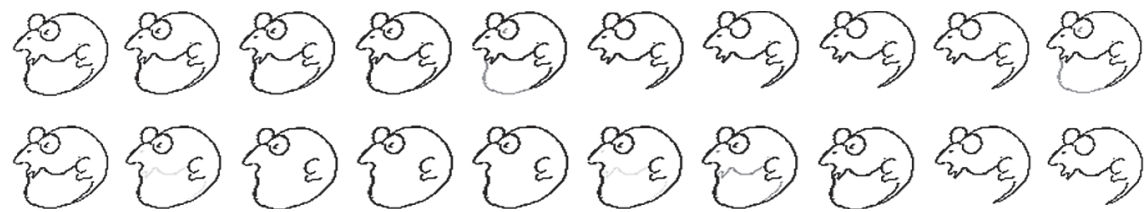

(b) A sequence of images taken from temporal associative memory

Fig. 7. The overlap states and the sequence of images over time. Upper images: the overlap curves show the state of associative memory at time $t$ with each of the corresponding memories. Lower images: temporal associative memory image sequence. The images are arranged according to the time course.

(a) The overlap states of 'man' and 'rat' perception with strong top-down feedback $(\mathrm{sc}=1.0)$

(b) A sequence of images taken from temporal associative memory

Two images were taken from Fig. 1 and resized to $78 \times 71$ images. Then, the images were bi-polarized with $1 \mathrm{~s}$ and $-1 \mathrm{~s}$ and used for training inputs. After the training phase, the system was tested with the test image that can be ambiguously perceived as man or rat.

It was assumed that the output of the integration units was initially determined by only bottom-up input in the interpretation phase. This is a reasonable assumption because no percept is activated until the system is instructed which temporal associative memory fits the stimulus. Therefore, we simply set all initial top-down inputs to 0s. As noted above, the output of the Widrow-Hoff network was used as input to temporal associative memory. At the beginning, the input $R_{j}$ was set to $a_{j}$, which means the initial internal representation is also determined by only the input from the previous stage. The whole updating cycle was repeated for 20 iterations in this simulation.

In this simulation, the top-down input (taken from the temporal associative memory) was multiplied by a scalar 1.0 in order to compare with the performance of the model when top-down influence was reduced (e.g. multiplied by 0.01 in the following simulation). We present the overlap of temporal associative memory in Fig. 7 (a), and the images taken 


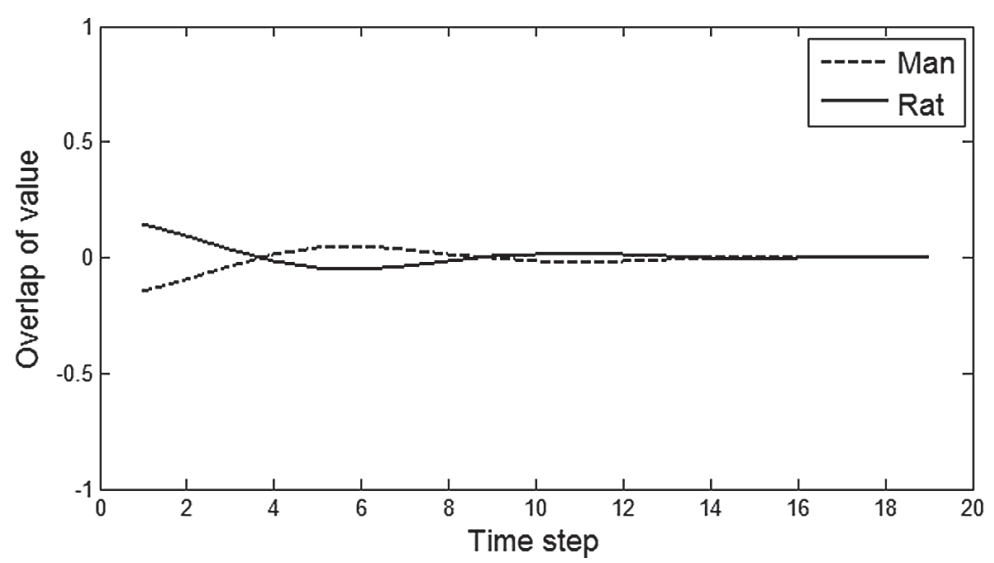

(a) The overlap states of 'man' and 'rat' perception with very weak top-down feedback $(\mathrm{sc}=0.01)$

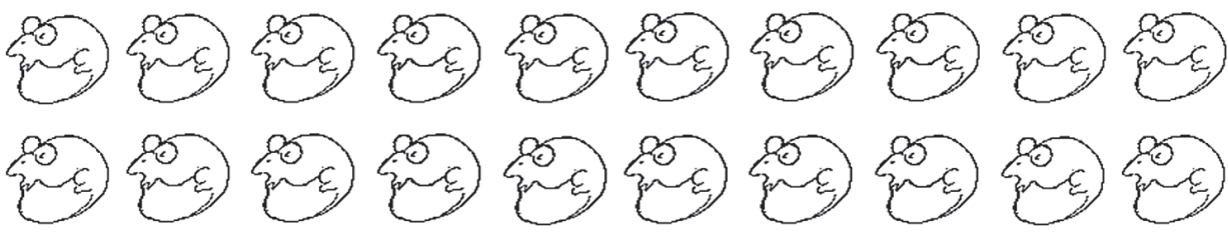

(b) A sequence of images taken from temporal associative memory

Fig. 8. The overlap states and a sequence of images taken form temporal associative memory. The topdown input was multiplied by a scalar -0.01 . The amplitude of overlap curve decreased to zero and the temporal associative memory remained almost unchanged.

(a) The overlap states of 'man' and 'rat' perception with very weak top-down feedback $(\mathrm{sc}=0.01)$

(b) A sequence of images taken from temporal associative memory

from the temporal associative memory in Fig. 7 (b) over the time course. As shown in both figures, the states corresponding to man and rat fluctuated from one state to the other along the time dimension. In the initial 4 time steps, the overlap curve oscillated with small amplitude, and did not reach to either 1 or -1 . However, as time went on, the overlap curve oscillated with larger amplitudes and reached near to 1 or -1 .

What would happen if top-down feedback was not properly delivered to the earlier processing stage? To answer this question, a simulation was carried out by manipulating top-down inputs. In this simulation, the top-down input was multiplied by a scalar value 0.01. The train and test procedure was the same as in the previous simulation except the manipulation of top-down inputs. The result is presented in Fig. 8, which shows that the fluctuation of overlap is damped over time. The initial amplitude of the overlap was diminished to zero, and the test image remained nearly unchanged. As noted earlier, strong top-down influence is needed in order to move the initial overlap state up (for "man") or down (for "rat"). However, the weak top-down influence (multiplied by a scalar 0.01) moved the next overlaps to zero state, and thus the overlap damply fluctuated. 


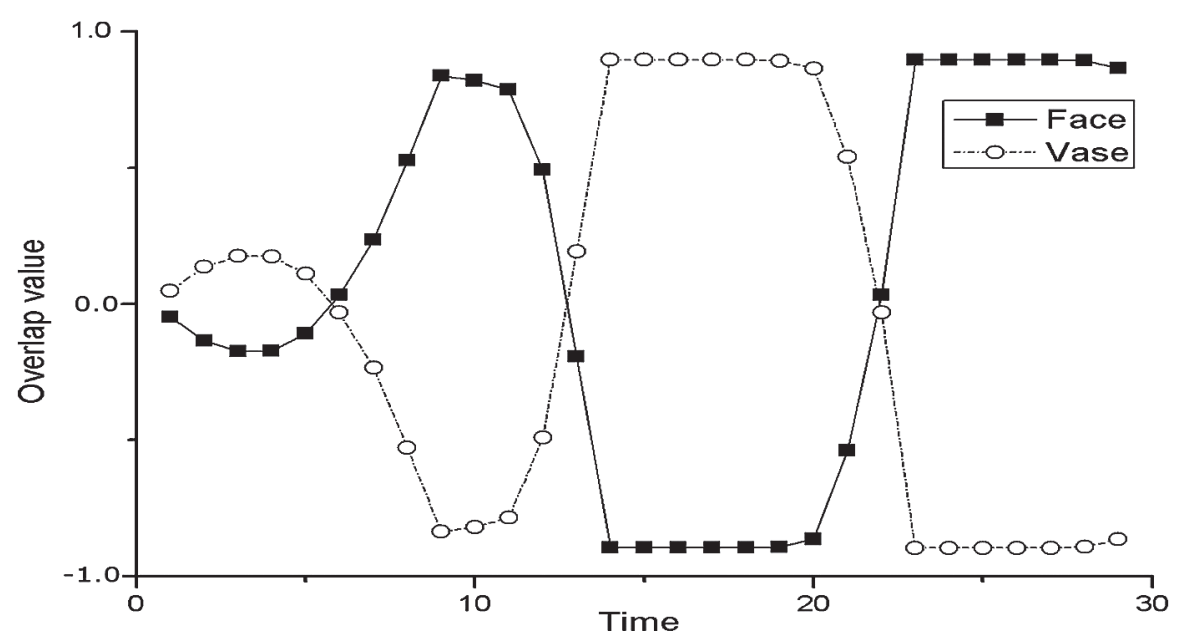

(a) The overlap states of 'Face-Vase' fluctuation
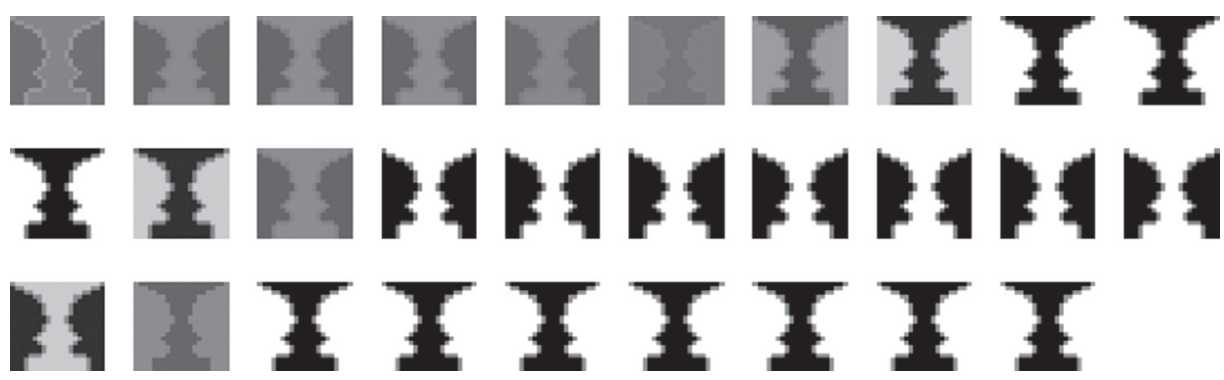

(b) Associative memory sequence driving the alternative perceptual states
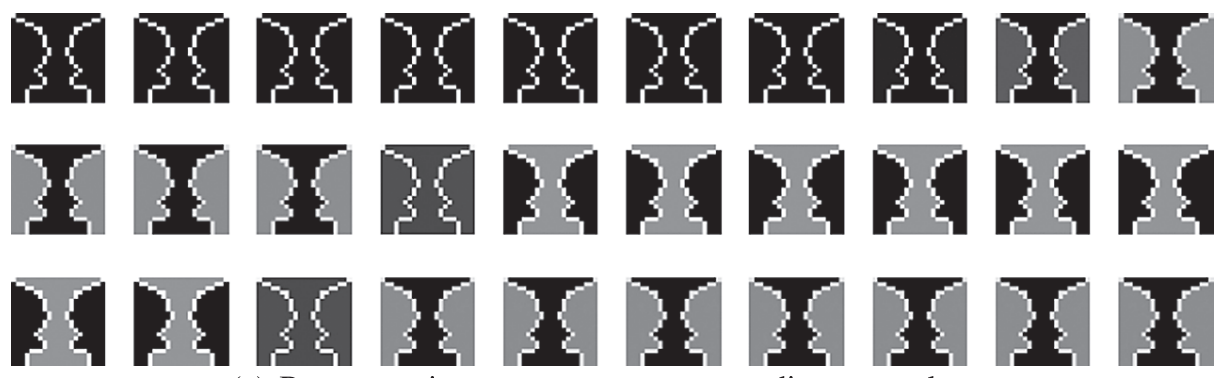

(c) Bottom-up image sequence corresponding to overlap states

Fig. 9. The curves show the state of associative memory at time $t$ with each of the corresponding memories. In this simulation, a step function was used for the kernel $G(t)$. The images are arranged according to the time course. The first image of bottom-up image sequence is the test image. The result shows the bottom-up image was dynamically changed over time course in accordance with temporal associative memory.

(a) The overlap states of 'Face-Vase' fluctuation

(b) Associative memory sequence driving the alternative perceptual states

(c) Bottom-up image sequence corresponding to overlap states 


\section{Simulation of Face-Vase Perceptual Reversal}

Another example is present in Fig. 9. The simulation was based on Rubin's figureground problem. Two images (consisting of $-1 \mathrm{~s}$ and $1 \mathrm{~s}$ ) - face and vase-were used for training inputs. The size of the image is 21 by 21 pixels. For computational convenience, we simply define white parts of the image as figure, while black parts are background. After the training phase, the system was tested with a boundary image that is shared by both training images.

We present the overlap of temporal associative memory, and the updated bottom-up images at different processing stages over the time course in Fig. 9. As shown in both figures, the states corresponding to face and vase dynamically fluctuated from one state to the other along the time dimension. The initial response of the integration units without top-down input is close to 0.5 . This can be considered as an ambiguous state since none of the units was strongly activated at the initial stage. When the system was in such an ambiguous state, neither configuration was dominantly perceived as figure or ground. However, when the system is near a certain stable state, the figure and ground became more clearly discriminated.

For example, when the overlap was polarized near 1 or -1 , the peak is considered as figure, whereas the trough is considered as background. As defined previously, the brighter parts of the image are the figure, while the darker parts of the image are the ground. Both configurations showed greater contrast when the overlap was polarized, rather than in the ambiguous state. Furthermore, the bottom-up image corresponds to the image of temporal associative memory, which clearly indicates that the bottom-up data was dynamically changed over the time course in accordance with temporal associative memory.

\section{DISCUSSION}

\section{Disambiguation of Figure-Ground Problem}

Due to the complex nature of the visual world, objects themselves are intrinsically ambiguous. For example, an object is occluded by others, visual events are not always continuously perceived, and there is a large number of variations in how a visual object may be seen in terms of shape, size, rotation, translation, feature properties etc. Evidence for analyzing the identification of a stimulus is given by the external visual world. Size, convexity and symmetry are among these sources of evidence, but other sources may be found in our internal knowledge from experience, such as meaningfulness, familiarity, expectation, and so on (Wagemans et al., 2012). How this evidence is integrated to separate a figure from its background is critically important to resolve ambiguity.

Many conventional theories that attempt to explain the figure-ground problem suggest that perceptual organization may occur prior to object recognition (Forsyth, Malik, Fleck, \& Ponce, 1997; Vecera \& O'reilly, 1998). At first glance, it seems reasonable to assume this because object recognition will be compounded with other objects in the absence of figure-ground separation. However, this assumption is contradicted by some phenomena such a more meaningful object being likely to be perceived as a figure rather than a 
meaningless object. In contrast to the conventional theory, Peterson (Peterson, 1994; Peterson \& Kim, 2001) argued that object representation can precede figure-ground separation. That is, a prefigural object representation may exist prior to the figure-ground organization.

Criticizing these serial processing views and making a compromise between two opposing explanations, an interactive approach in which bottom-up input interacts with top-down input in a hierarchical system was suggested (Domijan \& Setic, 2008; Vecera \& O'reilly, 1998). In this explanation, inputs from a lower layer continuously activate outputs in a higher layer, and activation from a higher layer provides feedback to units in a lower layer, allowing the model to converge to a stable state. Therefore, ambiguity caused by overall equivalence in terms of lower level cues - area, symmetry, convexity etc. - is resolved by top-down inputs that cooperate with bottom-up input. This idea is similar to our model.

\section{From One State to Another}

An interesting question brought up by Kawabata and Mori (1992) concerns initiation of a percept from viewing an ambiguous picture. Fixating on a part of an image - e.g., the beak or the mouth in the duck-rabbit picture - activates an interpretation. In other words, attending to one salient point may lead to one interpretation, and attending to another point may lead to a different interpretation. Spatial allocation of focal attention around an ambiguous picture contributes to a change from one percept to another (Pitts, Nerger, \& Davis, 2007; Slotnick \& Yantis, 2005).

In addition to spatial-based attention, object-based attention is also involved in perceptual alternation (Vecera, 2000). For example, patients with parietal lobe damage failed to report one of the pair of objects that was spontaneously presented and overlapped with the other. These balint patients were presented with stimuli in which a word and a picture were spatially overlapped. The patients failed to report the word even though they identified both the word and the picture when they were individually presented (Humphreys, Romani, Olson, Riddoch, \& Duncan, 1994). This case study implies that extinction of the word can be considered as a result of biased competition in favor of a picture since meaning is more quickly retrieved from a picture than a word. Even though we did not explicitly implement an attentional mechanism in the model, the feedback influences on the bottomup information, driven by temporal associative memory, can be considered as an attentive process. This process selectively modified the ambiguous bottom-up information in order to correspond to the internal representation biasing 'attention' to one of the set of interpretations.

As referred to earlier, the neural fatigue hypothesis puts the emphasis on automatic and spontaneous transition from one percept to another. This implies that perceptual fluctuation is a result of passive visual processing occurring on an early sensory level. This implication seems to be contradicted by itself because a percept representation does not simply involve sensory level processing; a set of neurons corresponding to a percept could be located in higher brain areas (Kleinschmidt, Büchel, Zeki, \& Frackowiak, 1998; Windmann, Wehrmann, Calabrese, \& Güntürkün, 2006). Moreover, the role of knowledge or learning about an ambiguous image showed that subjects failed to alternate their percepts 
if they were not explicitly informed that an ambiguous figure was reversible. This suggests that perceptual alternation is linked to voluntary control or at least higher level processing rather than sensory level processing.

However, it should be noted that there is much evidence that 'perceptual reversal' cannot be explained by top-down influence alone. For instance, the Marroquin pattern, composed of dots, involves the illusory appearance and disappearance of certain circles. Perceptual multi-stability can be accomplished by lateral competitive interaction between neuronal assemblies, rather than top-down influence (Feldman, 2003; Wilson, Krupa, \& Wilkinson, 2000). Cooperative interaction of neural units within an assembly would be needed to make a circle appear, whereas competitive interaction between assemblies would be needed to make another circle disappear.

\section{Brain Mechanism of Perceptual Reversal}

The underlying brain mechanisms for multi-stable perception have been studied using brain imaging techniques. Brain activities were measured during a perceptual transition and were compared to those activities associated with a stable perceptual state during multi-stable perception. These studies showed that attention-related frontal-parietal areas are responsible for initiating perceptual alternations by sending top-down signals to guide activity in the visual cortex (Leopold \& Logothetis, 1999). These top-down activations accentuate the neural representation of the perceived object in the early visual cortex (Tong, Wong, Meng, \& McKeeff, 2002). Deactivation occurred in the primary cortex and the pulvinar during the temporary absence of a dominant percept (Kleinschmidt et al., 1998). That is, perceptual fluctuation of ambiguous images involves many functionally and spatially segregated brain areas.

These brain imaging studies are compatible with our model that emphasizes the role of a high level of visual cognition, the integrative and cooperative operation between bottom-up and top-down information, and feedback modulation to the early stage during perceptual reversal (de Graaf, de Jong, Goebel, van Ee, \& Sack, 2011; Knapen, Brascamp, Pearson, van Ee, \& Blake, 2011; Sterzer et al., 2009). In our model, the transition between two alternatives is driven by temporal associative memory that provides feedback and topdown information to the lower layer. Minimizing the conflict caused by discrepancy between integration output and overlap values leads the system to a coherent perceptual state. Cooperation amongst the different processing modules is not only critically important to the transition from one percept to another, but also stabilizes the whole system into a perceptual experience.

\section{REFERENCES}

Aarts, E., \& Korst, J. 1989. Simulated annealing and boltzmann machines: A stochastic approach to combinatorial optimization and neural computing. New York: John Wiley \& Sons.

Atmanspacher, H., Bach, M., Filk, T., Kornmeier, J., \& Römer, H. 2008. Cognitive time scales in a Necker-Zeno model for bistable perception. The Open Cybernetics and Systemics Journal, 2, 234-251. Balcetis, E., Dunning, D., \& Granot, Y. 2012. Subjective value determines initial dominance in binocular 
rivalry. Journal of Experimental Social Psychology, 48, 122-129.

Blake, R., \& Wilson, H. 2011. Binocular vision. Vision Research, 51, 754-770.

Ciszak, M., Euzzor, M., Farini, A., Arecchi, F. T., \& Meucci, R. 2012. Modeling bistable perception with a network of chaotic neurons. Cybernetics and Physics, 1, 165-168.

Craft, E., Schütze, H., Niebur, E., \& von der Heydt, R. 2007. A neural model of figure-ground organization. Journal of Neurophysiology, 97, 4310-4326.

de Graaf, T. A., de Jong, M. C., Goebel, R., van Ee, R., \& Sack, A. T. 2011. On the functional relevance of frontal cortex for passive and voluntarily controlled bistable vision. Cerebral Cortex, 21, 2322-2331.

Ditzinger, T., \& Haken, H. 1989. Oscillations in the perception of ambiguous patterns a model based on synergetics. Biological Cybernetics, 61, 279-287.

Doherty, M. J., \& Wimmer, M. C. 2005. Children's understanding of ambiguous figures: Which cognitive developments are necessary to experience reversal? Cognitive Development, 20, 407-421.

Domijan, D., \& Setic, M. 2008. A feedback model of figure-ground assignment. Journal of Vision, 8, 1-27.

Ernst, B. 1986. Escher's impossible figure prints in a new context. In H. S. M. Coxeter, M. Emmer, R. Penrose, \& M. L. Teuber (Eds.), M. C. Escher: Art and Science (pp.125-134). Amsterdam: Elsevier Science.

Farid, H. 2002. Temporal synchrony in perceptual grouping: A critique. Trends in Cognitive Sciences, 6, 284-288.

Farid, H., \& Adelson, E. H. 2001. Synchrony does not promote grouping in temporally structured displays. Nature Neuroscience, 4, 875-876.

Feldman, J. 2003. What is a visual object? Trends in Cognitive Sciences, 7, 252-256.

Forsyth, D., Malik, J., Fleck, M., \& Ponce, J. 1997. Primitives, perceptual organization and object recognition (Tech. Rep.). University of California at Berkeley, Computer Science Division.

Gigante, G., Mattia, M., Braun, J., \& Del Giudice, P. 2009. Bistable perception modeled as competing stochastic integrations at two levels. PLoS Computational Biology, 5, e1000430.

Haykin, S. 1998. Neural networks: A comprehensive foundation (2nd ed.). Upper Saddle River, NJ: Prentice Hall.

Hopfield, J. J. 1982. Neural networks and physical systems with emergent collective computational abilities. Proceedings of the National Academy of Sciences, 79, 2554-2558.

Humphreys, G. W., Romani, C., Olson, A., Riddoch, M. J., \& Duncan, J. 1994. Non-spatial extinction following lesions of the parietal lobe in humans. Nature, 372, 357-359.

Kawabata, N., \& Mori, T. 1992. Disambiguating ambiguous figures by a model of selective attention. Biological Cybernetics, 67, 417-425.

Kay, J., \& Phillips, W. A. 1997. Activation functions, computational goals and learning rules for local processors with contextual guidance. Neural Computation, 9, 895-910.

Kleinschmidt, A., Büchel, C., Zeki, S., \& Frackowiak, R. S. 1998. Human brain activity during spontaneously reversing perception of ambiguous figures. Proceedings of the Royal Society B: Biological Sciences, 265, 2427-2427.

Knapen, T., Brascamp, J., Pearson, J., van Ee, R., \& Blake, R. 2011. The role of frontal and parietal brain areas in bistable perception. The Journal of Neuroscience, 31, 10293-10301.

Leopold, D. A., \& Logothetis, N. K. 1999. Multistable phenomena: Changing views in perception. Trends in Cognitive Sciences, 3, 254-264.

Long, G. M., \& Toppino, T. C. 2004. Enduring interest in perceptual ambiguity: Alternating views of reversible figures. Psychological Bulletin, 130, 748-768.

Lumer, E. D., Friston, K. J., \& Rees, G. 1998. Neural correlates of perceptual rivalry in the human brain. Science, 280, 1930-1934.

Palmer, S. E. 1999. Vision science: Photons to phenomenology. Cambridge, MA: MIT Press.

Peterson, M. A. 1994. Object recognition processes can and do operate before figure-ground organization. Current Directions in Psychological Science, 3, 105-111.

Peterson, M. A., \& Kim, J. H. 2001. On what is bound in figures and grounds. Visual Cognition, 8, 329-348.

Pitts, M. A., Nerger, J. L., \& Davis, T. J. 2007. Electrophysiological correlates of perceptual reversals for three different types of multistable images. Journal of Vision, 7, 1-14.

Riedel, U., Kühn, R., \& van Hemmen, J. L. 1988. Temporal sequences and chaos in neural nets. Physical Review A, 38, 1105-1108. 
Rock, I., Gopnik, A., \& Hall, S. 1994. Do young children reverse ambiguous figures? Perception, 23, 635644.

Rock, I., \& Mitchener, K. 1992. Further evidence of failure of reversal of ambiguous figures by uninformed subjects. Perception, 21, 39-45.

Rubin, E. 1915. Synsoplevede figurer. Copenhagen, Denmark: Gyldendalske.

Rubin, E. 1921. Visuell wahrgenommene figuren. Copenhagen, Denmark: Gyldendalske.

Rumelhart, D. E., Smolensky, P., McClelland, J. L., \& Hinton, G. E. 1986. Schemata and sequential thought processes in PDP models. In J. L. McClelland, D. E. Rumelhart, \& P. R. Group (Eds.), Parallel distributed processing: Explorations in the Microstructure of Cognition (Vol. 2, pp. 7-57). Cambridge, MA: MIT Press.

Slotnick, S. D., \& Yantis, S. 2005. Common neural substrates for the control and effects of visual attention and perceptual bistability. Cognitive Brain Research, 24, 97-108.

Sterzer, P., Kleinschmidt, A., \& Rees, G. 2009. The neural bases of multistable perception. Trends in Cognitive Science, 13, 310-318.

Tong, F., Wong, A., Meng, M., \& McKeeff, T. J. 2002. Brain areas involved in attentional control and perception of ambiguous figures. Journal of Vision, 2, 677a.

Vecera, S. P. 2000. Toward a biased competition account of object-based segregation and attention. Brain and Mind, 1, 353-384.

Vecera, S. P., \& O'reilly, R. C. 1998. Figure-ground organization and object recognition processes: An interactive account. Journal of Experimental Psychology: Human Perception and Performance, 24, 441-462.

Wagemans, J., Feldman, J., Gepshtein, S., Kimchi, R., Pomerantz, J. R., van der Helm, P. A., et al. 2012. A century of Gestalt psychology in visual perception: II. Conceptual and theoretical foundations. Psychological Bulletin, 138, 1218-1252.

Wilson, H. R., Krupa, B., \& Wilkinson, F. 2000. Dynamics of perceptual oscillations in form vision. Nature Neuroscience, 3, 170-176.

Windmann, S., Wehrmann, M., Calabrese, P., \& Güntürkün, O. 2006. Role of the prefrontal cortex in attentional control over bistable vision. Journal of Cognitive Neuroscience, 18, 456-471.

(Manuscript received 25 February, 2013; Revision accepted 8 January, 2014) 